\title{
Aliasing Detection and Reduction in Plenoptic Imaging
}

\author{
Zhaolin Xiao ${ }^{\dagger}$, Qing Wang ${ }^{\dagger}$, Guoqing Zhou ${ }^{\dagger}$, Jingyi $\mathrm{Yu}^{\ddagger}$ \\ ${ }^{\dagger}$ School of Computer Science, Northwestern Polytechnical University, Xi' an 710072, China \\ $\ddagger$ University of Delaware, DE 19176, USA
}

\begin{abstract}
When using plenoptic camera for digital refocusing, angular undersampling can cause severe (angular) aliasing artifacts. Previous approaches have focused on avoiding aliasing by pre-processing the acquired light field via prefiltering, demosaicing, reparameterization, etc. In this paper, we present a different solution that first detects and then removes aliasing at the light field refocusing stage. Different from previous frequency domain aliasing analysis, we carry out a spatial domain analysis to reveal whether the aliasing would occur and uncover where in the image it would occur. The spatial analysis also facilitates easy separation of the aliasing vs. non-aliasing regions and aliasing removal. Experiments on both synthetic scene and real light field camera array data sets demonstrate that our approach has a number of advantages over the classical prefiltering and depth-dependent light field rendering techniques.
\end{abstract}

\section{Introduction}

The availability of light field camera array and commercial plenoptic cameras has given rise to many solutions to traditionally challenging computer vision and graphics problems, ranging from multi-view stereo matching [27, $31,11]$, to panoramic synthesis $[29,28]$ and image matting [10]. A plenoptic camera is essentially a multi-view acquisition device with the goal to acquire discrete samples of the 4D light field. The camera baseline in the light field camera array $[28,25,26]$ is generally larger than the one in the light field camera such as Lytro [18] and Raytrix [23]. A unique capability of plenoptic camera is after-shot dynamic refocusing via wide-aperture filtering [8] or Fourier slicing [20]. However, the number of views (or the angular resolution) is often deemed insufficient to produce high quality refocused images. As a result, the refocused images will exhibit strong aliasing artifacts due to angular undersampling.

The cause of aliasing in light field refocusing has been thoroughly studied in both the spatial and frequency domains $[13,4,3,19]$. In the spatial domain, the aliasing

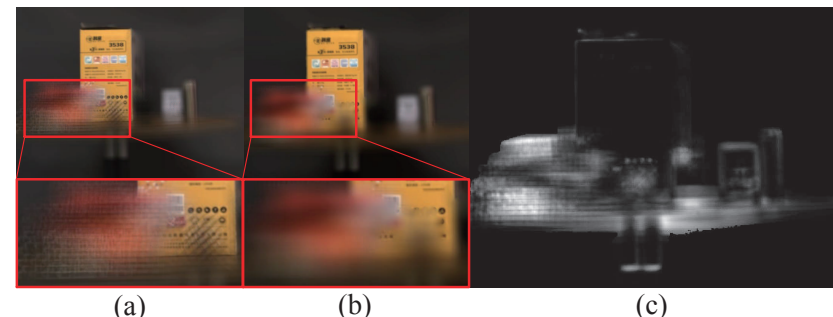

(a)

(b)

(c)

Figure 1. Angular Aliasing Detection and Reduction. (a) shows the classical light field refocusing result which exhibits severe aliasing. Our technique effectively detects the aliasing regions (c) and reduces aliasing to improve rendering (b).

artifacts occur at the out-of-focus regions and are attributed to insufficient number of ray samples. To reduce aliasing, prefiltering [13] can be used to reduce the spatial artifacts. In the frequency domain, Chai et al. [4] presented a comprehensive analysis on the tradeoff between sampling density and depth resolution. They further suggested that a sufficient condition to avoid aliasing artifacts is to limit the disparity of all scene elements to \pm 1 pixel. Further, one can minimize aliasing by positioning the geometry proxy plane [8] at the depth that corresponds to the average of the minimum and maximum disparity.

In reality, implementing the sufficient aliasing-free condition is difficult. To ensure the disparity less than one pixel, the camera/microlens baseline should be ultra small, and often even smaller than the camera/microlens sizes. The condition is not necessary either. Consider a light field of a constant color wall. Even if the light field is severely undersampled, the refocused results will not exhibit aliasing. In contrast, if the wall is highly textured, the refocused image will exhibit aliasing and the aliasing pattern depends on the wall texture and the sampling pattern. This implies that a scene-dependent analysis is needed to properly characterize aliasing.

Our work is also motivated by the need for improving the visual quality in the refocused rendering. Reducing aliasing using a denser microlens array will reduce the effective image resolution. For example, in Lytro, the effective resolution is 0.7 megapixel even using a 11 megapixel sensor. In 
fact, balancing between the spatial and angular resolution is still an open problem in light field imaging [7]. Recent solutions $[2,6]$ that first recover scene depth and then use it in rendering have shown promising results. However, reliable scene geometry estimation via stereo matching [27, 31, 11] or volumetric reconstruction [5] is still difficult.

In this paper, we present a different solution that first detects and then removes aliasing at the light field refocusing stage. Specifically, we reconstruct a set of refocused images by randomly selecting/excluding certain angular views. We then compare the coefficient of variation of reconstructed scene points, with high-variance points indicating aliasing. For the aliasing regions, we use lower-frequency terms of the decomposition for reconstructing the refocused image. Experiments on both synthetic scene and real light field camera array data sets demonstrate that our approach has a number of advantages over the classical prefiltering and depth-dependent light field rendering techniques.

\section{Related work}

Modeling and reducing aliasing in light field rendering is a long term problem in image-based rendering. The recent commodity light field cameras have renewed the interest on exploring the problem. Earlier approaches rely on light field prefiltering that can implement either physically by using a wide aperture camera or computationally by first oversampling the light field and then applying a low-pass filter [13]. Prefiltering can also be combined with dynamic light field reparameterization to reduce aliasing at any focal depth [8]. The prefiltering technique can effectively reduce aliasing but will also introduce excessive blur in the refocused image, especially when the light field is undersampled. Stewart et al. [24] compensated over-blurring by combining multiple linear filters to simultaneously reduce aliasing and maintain image sharpness. Zwicker et al. [33] alleviated aliasing in $3 \mathrm{D}$ displays by interpolating more views than what the display acquires. $\mathrm{Ng}$ [20] suggested that the spatial domain rendering and aliasing reducing algorithms can be more efficiently implemented in the frequency domain by band limited filtering and slicing.

With the availability of the commodity light field cameras such as Lytro [18] and Raytrix [23], one can dynamically control the angular sampling depending on scene composition, desired photographic effects, etc. The Raytrix and the Adobe plenoptic can dynamically change the microlensto-sensor space for trading between spatial and angular resolutions [22, 17, 7]. However, due to limits on sensor sizes/ resolution and microlens baselines, generating a high spatial resolution image has to sacrifice the angular resolution. As a result, the aliasing artifacts at the out-of-focus regions can be severe in the focused plenoptic camera [17], even with smart image demosaicing [32]. It is also possible to use depth-dependent light field rendering [16] to reduce alias- ing. However, these techniques require solving the scene reconstruction problem, which is traditionally challenging and slow.

Light field cameras can also be implemented using coded apertures. Liang et al. [14] developed a programmable aperture photography system that can obtain a full resolution light field via view-dependent depth estimation. Bishop et al. [2] introduced an anti-aliasing filter that also incorporates multi-view depth information. Levin et al. [12] have shown that, if scene depth information is known, one can use mixture-of-Gaussians derivative priors to recover a nearly aliasing-free light field. All these techniques attempt to avoid aliasing before light field rendering whereas we aim to detect potential aliasing regions and then reduce aliasing at the rendering stage.

\section{Angular Aliasing Analysis and Detecting}

We start with studying the cause of aliasing in light field imaging in the spatial domain. For clarity, we focus our analysis on light field camera array in which the angular sampling is generally sparse due to the large camera baseline. The analysis can be applicable to plenoptic cameras such as Lytro and Raytrix by mapping each microlens to a pinhole camera in the array.

\subsection{Aliasing in Refocusing}

The digital refocusing technique using the light field data is commonly referred to as synthetic aperture photography $[8,25]$. In general, synthetic aperture produced by the camera array is much larger than the one produced by Lytro or Raytrix. We assume each constituent camera in the array is pinhole in which each ray represents an angular sample of the scene. To synthetically focus on an arbitrary focal surface, one can query and then integrate corresponding rays from all cameras, similar to gathering rays using a thin-lens with a wide aperture, as illustrated in Figure 2.

Conceptually, the main difference between synthetic and real aperture imaging is that, the real one acquires all light rays passing through the camera whereas the synthetic one

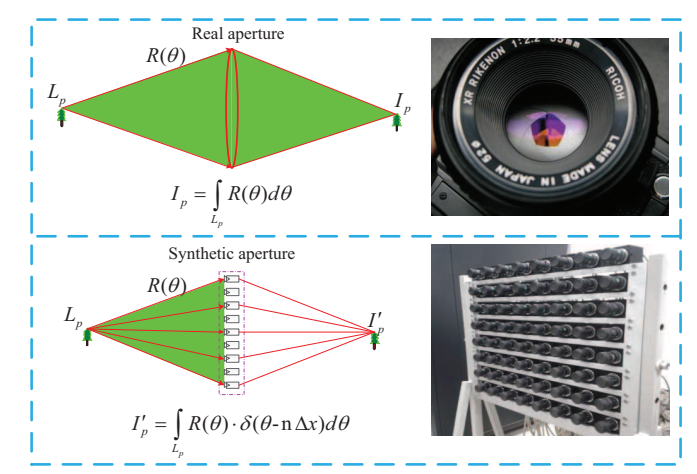

Figure 2. Refocusing Using a Real vs. Synthetic Aperture. 
only gathers a subset of rays, i.e., ray samples. Therefore, the synthetic aperture case can be viewed as a sampled version of the thin-lens system. Let $L_{p}$ be the complete set of incident rays from a 3D space point $p$ and $R(\theta)$ be a ray of $L_{p}$ with angle $\theta$. The real aperture image $I_{p}$ is represented as $I_{p}=\int_{L_{p}} R(\theta) d \theta$. In the camera array case, the synthetic image $I_{p}^{\prime}$ is,

$$
\begin{aligned}
I_{p}^{\prime} & =\int_{L_{p}} R(\theta) \delta(\theta-n \Delta x) d \theta \\
& =\sum_{L_{p}} R(\theta)-\sum_{L_{p}} R(\theta) \bar{\delta}(\theta-n \Delta x)
\end{aligned}
$$

where $\delta(\cdot)$ is a Dirac's delta function and $\Delta x$ is the sampling interval, $n \in \mathbb{N}$. If taking the sampling noise $\varepsilon$ into consideration, the relationship between $I_{p}^{\prime}$ and $I_{p}$ is

$$
I_{p}^{\prime}=I_{p}-I_{p} * \bar{\delta}(\theta-n \Delta x)+\varepsilon .
$$

Eqn.(2) reveals that aliasing is caused by $I_{p} * \bar{\delta}(\theta-n \Delta x)$. If we know the camera array setting, we can derive a maximum aliasing-free sampling interval $\Delta x^{*}$ (or a minimum sampling rate $\left.S_{x^{*}}\right)$, i.e., to any sampling interval $\Delta x<$ $\Delta x^{*}$, the term $I_{p} * \bar{\delta}(\theta-n \Delta x)$ could be negligible. For simplicity, we denote $S_{x^{*}}=\frac{1}{\Delta x^{*}}$ and $S_{x}=\frac{1}{\Delta x}$. The aliasing artifact hence is determined by the sampling ratio $R$,

$$
R= \begin{cases}\frac{S_{x}}{S_{x^{*}}}<1 & \text { if aliasing } \\ \frac{S_{x}}{S_{x^{*}}} \geq 1 & \text { if non-aliasing }\end{cases}
$$

Next, we employ the classical two paralleled-plane model [13] and analyze the relationship between $S_{x^{*}}$ and $S_{x}$ in the 2D light field space [15]. As shown in Figure 3, all rays originating from an arbitrary surface are parameterized by the camera plane $V$ and image plane $T$. On the camera

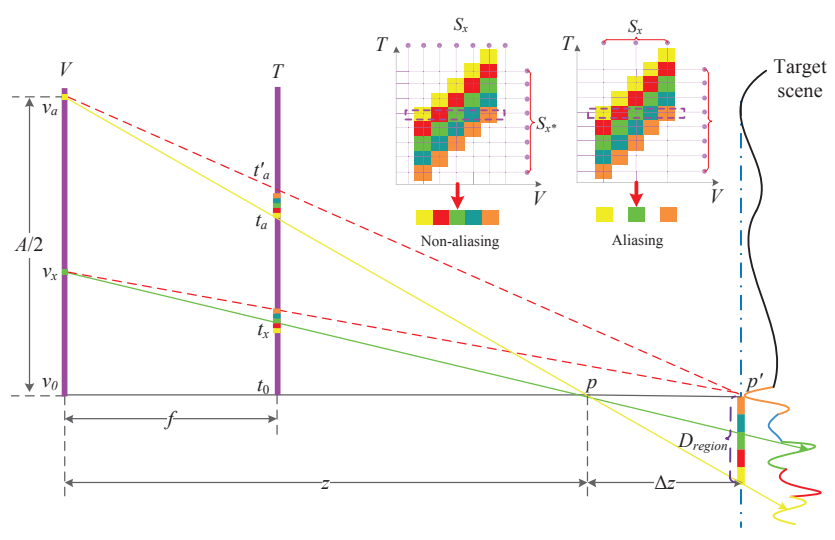

Figure 3. Angular Undersampling and Aliasing. If the camera and image planes have the same sampling rate, the refocusing results should be free of angular aliasing. plane $V, 1 / \Delta x$ is equivalent to the number of cameras. We choose the central camera $v_{0}$ as the reference one. Assume all cameras focus at a specific 3D point $p$ whose depth is $z$. If $p$ is not a real physical point in the space, all rays passing through $p$ can be traced back to the actual surface $(\Delta z$ away from $p$ ). We mark this region $D_{\text {region }}$ in color. The boundary of $D_{\text {region }}$ can be determined by two lines of $\overline{v_{0} p}$ and $\overline{v_{a} p}$, where $v_{a}$ represents the outermost camera on $V$.

Assume that the scene is Lambertian and camera array is uniformly distributed, for each camera $v_{x}$ within $v_{0}$ and $v_{a}$, to sample $t_{x}$ is equivalent to sampling between $t_{a}$ and $t_{a}^{\prime}$ in camera $v_{a}$. If camera counts between $v_{0}$ and $v_{a}$ is less than the pixel counts between $t_{a}$ and $t_{a}^{\prime}$, the aliasing artifacts will appear perceivably, as shown in the top right of Figure 3. On the image plane $T, t_{a}$ and $t_{0}$ is a pair of correspondence of $p$ in camera $v_{a}$ and $v_{0}$ respectively. Thus we have,

$$
t_{a}=t_{0}-\frac{f}{z}\left(v_{a}-v_{0}\right)=t_{0}-\frac{f}{z} \frac{A}{2}
$$

where $A$ is the aperture size.

From the similitude relationship in Figure 3, we can derive $\left|t_{a}-t_{a}^{\prime}\right|$ as,

$$
\left|t_{a}-t_{a}^{\prime}\right|=f \frac{A}{2} \frac{\Delta z}{z^{2}+z \Delta z}
$$

Therefore, the expected sampling interval $\Delta x^{*}$ can be derived as $1 /\left|t_{a}-t_{a}^{\prime}\right|$. Regarding $\alpha_{t}$ as the frequency of the texture on the image plane $T$, we can derive $R$ as,

$$
R=\frac{S_{x}}{S_{x^{*}}}=\frac{S_{x}}{\alpha_{t}\left|t_{a}-t_{a}^{\prime}\right|}=\rho \frac{1}{f} \frac{z^{2}+z \Delta z}{\Delta z} \frac{1}{\alpha_{t}}
$$

where $\rho=\frac{S_{x}}{A / 2}$ denotes the sampling density on the camera plane. The term of $\rho \frac{1}{f}$ is the property of camera array while $\frac{z^{2}+z \Delta z}{\Delta z}$ and $\frac{1}{\alpha_{t}}$ depend on the scene geometry and texture.

It is important to note that our analysis is different from the frequency aliasing analysis [4] in a number of ways. [4] explains if aliasing could be aliasing but neither guarantees that aliasing would occur or reveal where in the image it would occur. In contrast, our derivation explicitly states which part of the image will exhibit aliasing. Second, [4] derives the sufficient condition on aliasing-free rendering in the narrow aperture case (e.g., bilinear interpolation for view synthesis) whereas we derive the necessary sampling ratio to guarantee aliasing free rendering in the wide aperture (refocusing) filter. In particular, our analysis reveals that the aliasing-free sampling rate $S_{x^{*}}$ is scene geometry and texture dependent, which is the first explicit derivation that correlates aliasing with scene composition in the spatial domain.

Eqn.(6) shows that there are four cases that angular aliasing would be minimum. 
1) $\Delta z=0$. In this case, the focal plane coincides with actual scene geometry and the sampling rate $S_{x}$ is always sufficient.

2) $S_{x} \rightarrow+\infty$ or $A \rightarrow 0$. In this case, the sampling density $\rho \rightarrow+\infty$. For example, imaging using a real thinlens or using a pinhole camera will be aliasing free.

3) $f \rightarrow 0$. If the plane $V$ and $T$ are close enough, $\left|t_{a}-t_{a}^{\prime}\right|$ can be extremely small. Thus, the angular aliasing can be avoided due to the low resolution of rendering image.

4) $\alpha_{t} \rightarrow 0$. If the scene is textureless or the texture is highly smooth (very low frequency), the refocused results will not produce major aliasing at the out-of-focus regions.

If both scene geometry and texture are known, one can handle aliasing reduction at the rendering stage. For example, the depth-dependent rendering methods [30] assume that $\Delta z$ is known in Eqn.(6) and can estimate the size of the defocus blur kernel for conducting spatial blurs to emulate angular blurs. However, these techniques require depth estimation. In Section 3.3, we present a depth-free aliasing reduction scheme purely based on adaptive sampling.

\subsection{Aliasing Detection}

Recall that for a given scene within a finite range of distance, to a specific rendering point $p, S_{x^{*}}$ is a constant while $R$ would vary with $S_{x}$. We denote $U$ as all possible imaging results of points $p, U=\left\{I_{p}^{\prime}\left(S_{x}\right) \mid S_{x} \in[0,+\infty)\right\}$, denote $\Omega$ as all angular aliasing results $\Omega=\left\{I_{p}^{\prime}\left(S_{x}\right) \mid S_{x} \in\right.$ $\left.[0,+\infty), S_{x}<S_{x^{*}}\right\}$, and $\bar{\Omega}$ the possible over-sampled conditions. Obviously, $U=\Omega \cup \bar{\Omega}$ and $\Omega \cap \bar{\Omega}=\varnothing$. As shown in Figure 4, the red shaded area $\Omega$ corresponds to aliasing sampling conditions, whilst the blue shaded area represents aliasing-free sampling conditions. By Eqn.(3), $R>1$ iif $I_{p}^{\prime}\left(S_{x}\right) \in \bar{\Omega}$ and the aliasing term $I_{p} * \bar{\delta}(\theta-n \Delta x)$ is near zero. In this case, we have the following corollary.

Corollary: $\forall S_{x_{i}}, S_{x_{j}} \geq S_{x^{*}}$ corresponds to $I_{p}^{\prime}\left(S_{x_{i}}\right)$ and $I_{p}^{\prime}\left(S_{x_{j}}\right) \in \bar{\Omega}$, then $\left|I_{p}^{\prime}\left(S_{x_{i}}\right)-I_{p}^{\prime}\left(S_{x_{j}}\right)\right| \leq 2 \varepsilon$.

In particular, when $S_{x_{i}}=S_{x_{j}}$ and $S_{x_{i}} \in \bar{\Omega}$, the corollary still holds, i.e., all possible observations $I_{p}^{\prime}\left(S_{x_{i}}\right)$ (here

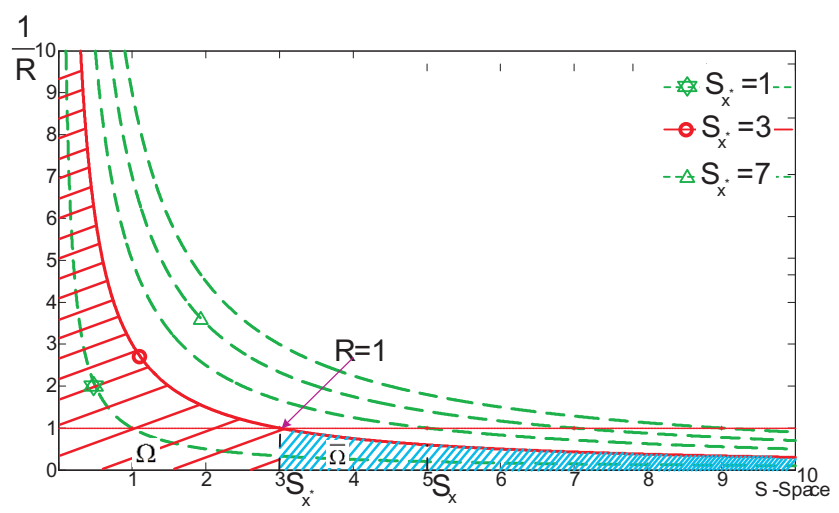

Figure 4. An Illustration of Our Sampling Rate Space. $x_{i}$ refers to different sampling pattern with the same sampling rate) will appear similar. In this case, aliasing detection is equivalent to solving the following problem.

Aliasing detection: For a given $S_{x_{0}}$, if $\exists\left(S_{x_{i}}, S_{x_{j}}\right)$, satisfying $S_{x_{i}}, S_{x_{j}} \leq S_{x_{0}}$ and $\left|I_{p}^{\prime}\left(S_{x_{i}}\right)-I_{p}^{\prime}\left(S_{x_{j}}\right)\right|>2 \varepsilon$, then $I_{p}^{\prime}\left(S_{x_{0}}\right) \in \Omega$.

If we set $S_{x_{0}}=S_{x^{*}}$, there is only one sampling pattern, i.e., the full aperture condition. Consequently, we cannot directly apply the aliasing detection scheme without altering the distribution of camera array. Therefore, we need to slightly relax the lower bound of the sampling requirement from $S_{x_{0}}$ to $(1-\gamma) S_{x_{0}}$, where $\gamma$ is a relax factor.

Let $P_{\gamma}\left(S_{x_{0}}\right)=\left\{S_{x} \mid(1-\gamma) S_{x_{0}} \leq S_{x} \leq S_{x_{0}}\right\}$ denote the new sampling rate space. The cardinality of $P$ is $\sum_{n=(1-\gamma) S_{x_{0}}}^{S_{x_{0}}} C_{S_{x_{0}}}^{n}$. However, it will be too expensive to compare arbitrary $I_{p}^{\prime}\left(S_{x_{i}}\right)$ and $I_{p}^{\prime}\left(S_{x_{j}}\right)$ when $S_{x_{0}}$ is large. We therefore randomly choose $N$ samples from $P$ to form a subset of observation images $M=\left\{I_{p}^{\prime}\left(S_{x_{i}}\right) \mid S_{x_{i}}=\right.$ $\left.\operatorname{random}_{i}\left(P_{\gamma}\left(S_{x_{0}}\right)\right), i=1, \ldots, N\right\}$. We then apply aliasing detection on $M$ as an approximation on $P$. To further reduce the computational cost, we introduce the coefficient of variation $\mathcal{C}_{v}$ as a metric to detect the distribution of $M$. We choose this $\mathcal{C}_{v}$ as an aliasing metric in accordance with Weber's law [9], since aliasing is not only determined by the intensity variations but also by the base of intensity.

$$
\mathcal{C}_{v}=\frac{\sigma}{\mu}=\frac{\sqrt{\frac{1}{N} \sum_{i=1}^{N}\left(I_{p}^{\prime}\left(S_{x_{i}}\right)-\mu\right)^{2}}}{\mu}
$$

where $\mu$ is the mean of SAIs under different sampling rates or patterns. $\mathcal{C}_{v}$ is close to zero when $I_{p}^{\prime}\left(S_{x_{i}}\right) \in \bar{\Omega}$, as revealed by the corollary. Otherwise, $\mathcal{C}_{v}$ will increase as the number of $I_{p}^{\prime}\left(S_{x_{i}}\right) \in \Omega$ increases. If the observed $\mathcal{C}_{v}$ is greater than a given threshold $T$, we regard the aliasing condition is satisfied, such that $I_{p}^{\prime}\left(S_{x_{0}}\right) \in \Omega$. In general, our aliasing detection is concluded in Algorithm 1.

It is important that our algorithm needs to slightly relax the sampling space by removing some angular samples randomly. We assume this slight relaxation will not affect the aliasing artifacts. In practice, we have to face a tradeoff between scheme effectiveness and robustness. In Section 4, we will further discuss how relaxation affects false positive and false negative in aliasing detection. The image quality can be significantly improved with a proper selected $\gamma$, especially when using the camera array system.

\subsection{Aliasing-Reduction in Refocusing}

Once we have aliasing-detection result, we can conduct aliasing-reduction at the light field refocusing stage. Recall that to implement Algorithm 1, we need to generate a collection of synthetic aperture images (SAIs). This can be achieved by randomly blocking some constituent cameras as different sampling patterns. The SAIs are synthesized by 


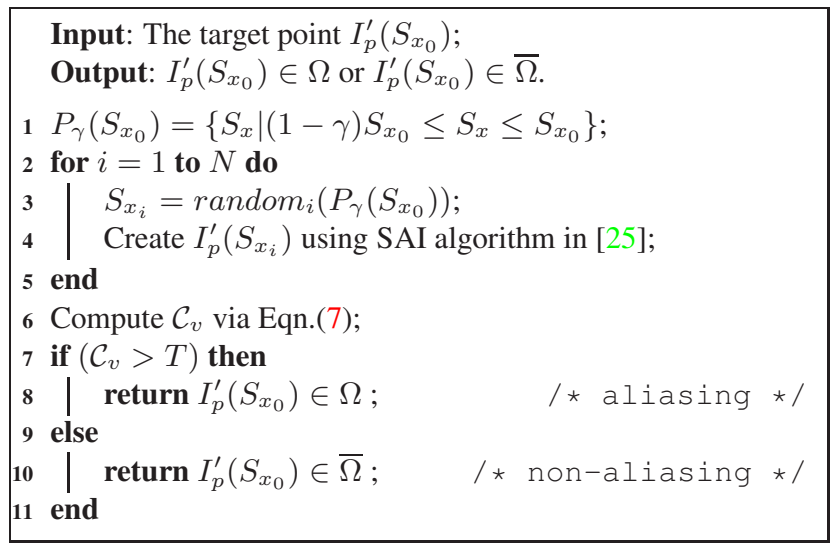

Algorithm 1: Aliasing Detection in Refocusing Stage.

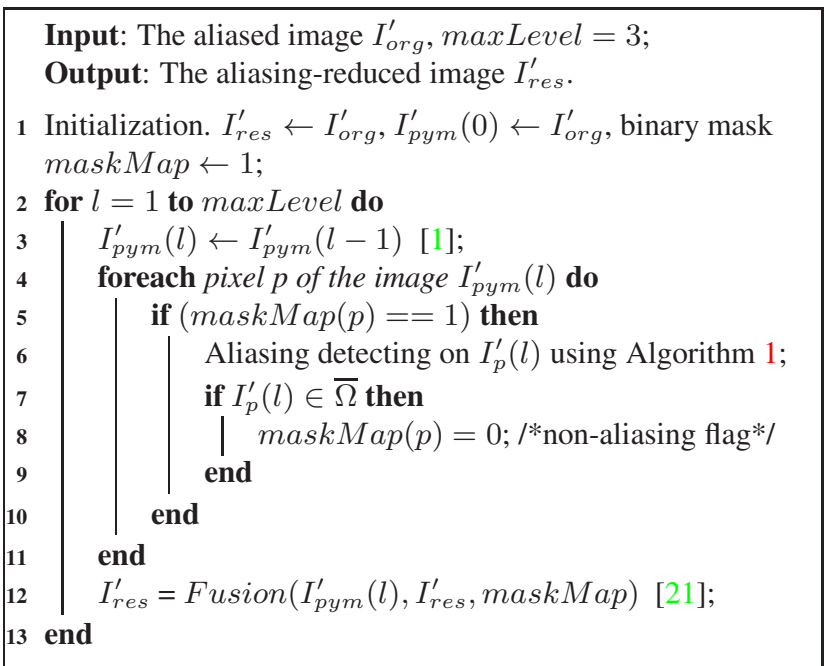

Algorithm 2: Aliasing Reduction in Light Field Refocusing.

employing the algorithm in [25]. As mentioned above, the angular aliasing can be significantly alleviated by decreasing resolution on image plane. Thus, we build a Gaussian pyramid of the SAIs [1], so that aliasing artifacts will be less significant at higher pyramid levels. The key idea here is to replace the aliasing region with non-aliasing ones extracted from images at high pyramid level. To decide the target region, we apply aliasing detection on SAIs at different level of pyramid. For each image point, we denote $l^{*}$ as the minimum pyramid level, on which the image point meets non-aliasing condition $\mathcal{C}_{v} \leq T$.

$$
\begin{aligned}
& \quad l^{*}=\min (l, \max \text { Level }) \\
& \text { s.t. } g(l)=\mathcal{C}_{v} \leq T
\end{aligned}
$$

where $l$ is a pyramid level, maxLevel is the pyramid maximum level, and $g(\cdot)$ is the aliasing detection function.

The simplest approach is to replace the aliasing region with non-aliasing template directly. However, directly replacing the pixel can cause severe seaming boundaries prob-

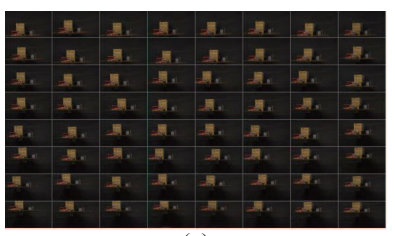

(a)

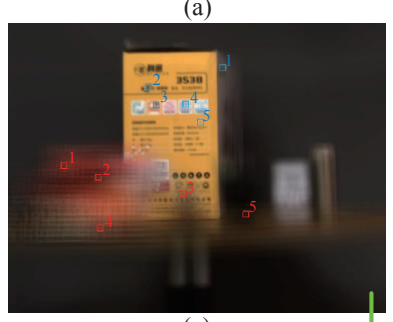

(c)

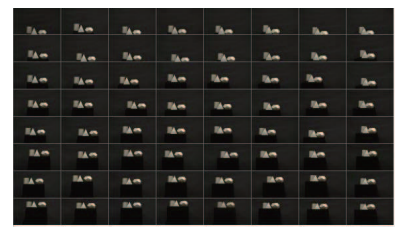

(b)

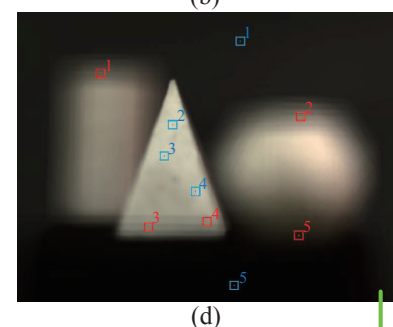

(d)

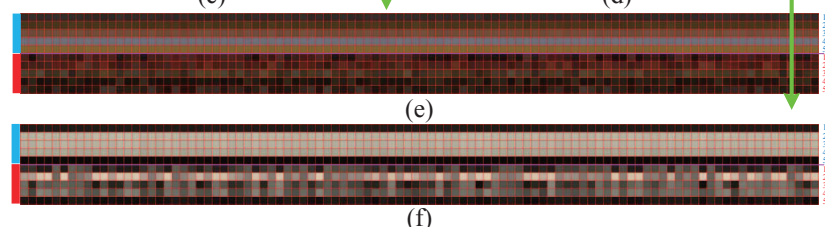

(f)

Figure 5. Comparisons of aliasing vs. aliasing-free pixels in different synthetic aperture images. (a) and (b) show the multiview data acquired with a camera array. (c) and (d) show the traditional light field refocusing images. (e) and (f) show the refocused results with 100 different sampling patterns.

lem. Therefore, we conduct a gradient domain fusion process [21]. We stitch different image regions by their gradients and then solve for the Poisson equation. The complete aliasing-reduction algorithm is summarized in Algorithm 2.

\section{Experimental Results}

All experiments are conducted on the light field data acquired by the $8 \times 8$ camera array in which angular aliasing is most severe. The elemental CCD camera (CK-IH046C) has a $752 \times 576$ resolution and $37.0^{\circ}$ field of view. The baseline between two adjacent cameras is $70 \mathrm{~mm}$, as shown in Figure 2. As shown in Figure 5(a)-(b), each sub-image is captured by an element camera in the array. Due to the large baseline between cameras, the acquired light fields are undersampled in the angular domain. Generating the SAIs using traditional interpolation and integral techniques [25] results in severe aliasing, as shown in Figure 5(c)-(d).

Section 3.2 has revealed that the aliasing artifacts depend heavily on the sampling patterns. Using different patterns, the SAIs exhibit significantly different aliasing structures. In contrast, the aliasing-free points remain nearly the same despite pattern changes. For better illustration, we select several typical pixels and show their variations using different sampling patterns in Figure 5(e)-(f). For example, the blue aliasing-free points have coherent appearance whereas the red aliased points apparently exhibit large variations under different sampling patterns. 

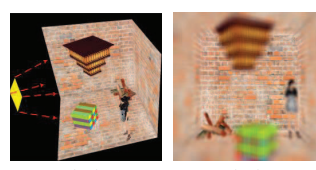

(a2)
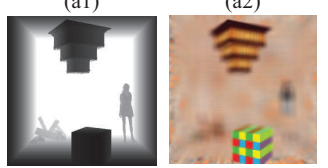

(b1)
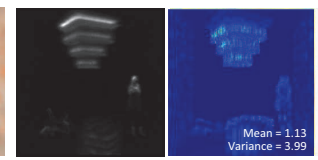

(a4)

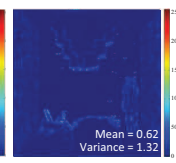

(a5)

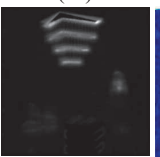

(b3)

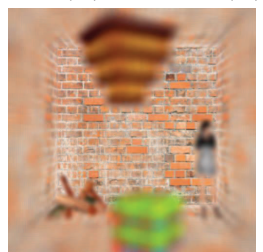

(c1)

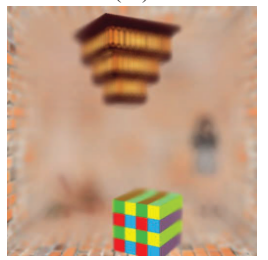

(d1)

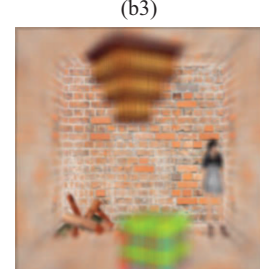

(c2)

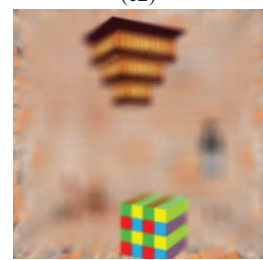

(d2)

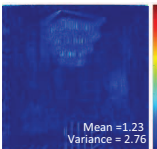

(b4)

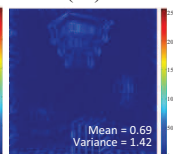

(b5)

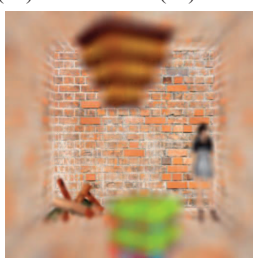

(c3)

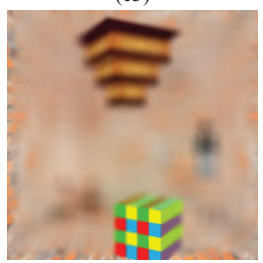

(d3)
Figure 6. Comparisons of different aliasing reduction techniques on a synthetic data set.

To validate our aliasing detection and reduction algorithms, we first generate a synthetic light field by rendering an OpenGL scene, as shown in Figure 6(a1). In front of a Brickwall room, we synthesize an $8 \times 8$ equidistant camera array, which is $5 \times 5$ units large in size and is 40 units from the back wall. For each camera, we generate a $512 \times 512$ resolution picture with $45^{\circ}$ field of view. Through the experiments (see more details in Figure 8 and Figure 9), we select $\gamma=0.2$ and $N=100$, which can obtain the best results. We set $T=0.02$ which corresponds to the minimal intensity of perceivable aliasing. Any potential aliasing below this level will be ignored and viewed as noise.

We conduct our algorithms on the synthetic scene to verify their effectiveness, as shown in Figure 6. Figure 6(a1) shows an artificial scene with known depth (b1). Given two different focused planes (a2) at background wall and (b2) at the front of Rubick-cube, we obtain the aliasing maps (a3) and (b3) through our detection algorithm. Based on known depth, the baseline defocused rendering results are shown in (c1) and (d1) by using the depth-aware rendering [30]. Another set of results (c2) and (d2) are rendered using prefiltering [13]. The rendered results using our method are shown in (c3) and (d3) respectively. We observe that our results better preserve sharp edges in the focused regions and effectively reduce aliasing in the defocused regions. In contrast, the prefiltering results exhibit excessive blurs in the focused regions, e.g., the brick wall in (c2) and the Rubick-cube in (d2). Taking depth-aware rendering $(\mathrm{c} 1, \mathrm{~d} 1)$ as a baseline, its gradient map differences with prefiltering rendering and ours are shown in $(\mathrm{a} 4, \mathrm{~b} 4)$ and $(\mathrm{a} 5, \mathrm{~b} 5)$ respectively. Our approach exhibits slight difference in the defocused regions with respect to depth-aware rendering method. However, it is important to note that our approach is depth-free and the visual quality is comparable. According to the mean and variance of gradient differences, our approach obviously outperforms the profiltering method, which apparently preserves more details in the focused regions.

In Figure 7, we demonstrate our technique using the real camera array. For each data set, we experiment our algorithms at two focal depths. Compared with Figure 7(a), the aliasing artifacts using our solution are significantly reduced in Figure 7(c). At the same time, the focused high frequency regions are well preserved. The prefiltering algorithm effectively reduces aliasing but introduces blurs in the focused regions, as shown in Figure 7(b). Figure 7(d) shows the closeup views on the details in red and blue boxes of (a)(c). Figure 7(e) shows the aliasing detection results at the full resolution of the original image in which the intensity corresponds to $\mathcal{C}_{v}$ in our aliasing detection process.

In Figure 8, we plot the aliasing detection results with respect to parameter $N$ (the number of sampling patterns) from 20 to 150 . We observe that the detection is more stable with a large $N$. For example, $N \geq 100$ is sufficient for an $8 \times 8$ camera array. The relaxation factor $\gamma$ determines the upper bound of $N$. However, we cannot set $\gamma$ arbitrarily large since relaxation in the sampling space can introduce new aliasing frequency and cause false positives in our detection. Therefore, we generally need to tradeoff between detection accuracy and robustness. In Figure 9, we show two examples for illustration. Group 2 is falsenegative-detection and group 4 is false-positive-detection. For our camera array system, $\gamma=0.2$ is sufficient as shown in group 3.

\section{Conclusion and Future Work}

We have presented a new aliasing detection and reduction scheme for light field refocusing. Our analysis is based on spatial-domain analysis that directly associates aliasing with scene geometry and texture. To detect aliasing, we reconstruct a set of refocused images where certain angular views are randomly selected/excluded, hence simulating a random programmable aperture. We then compare the coefficient of image variation across these apertures for detecting aliasing. Once we detect aliasing, we apply a multiscale gradient fusion technique that replaces the aliased regions with aliasing free ones.

There are a number of future directions we plan to explore. Our experiments are restricted to the camera array where the camera baseline is large and aliasing is more problematic. An immediate future step is to apply our algorithm on the Lytro and Raytrix cameras. Since angular 

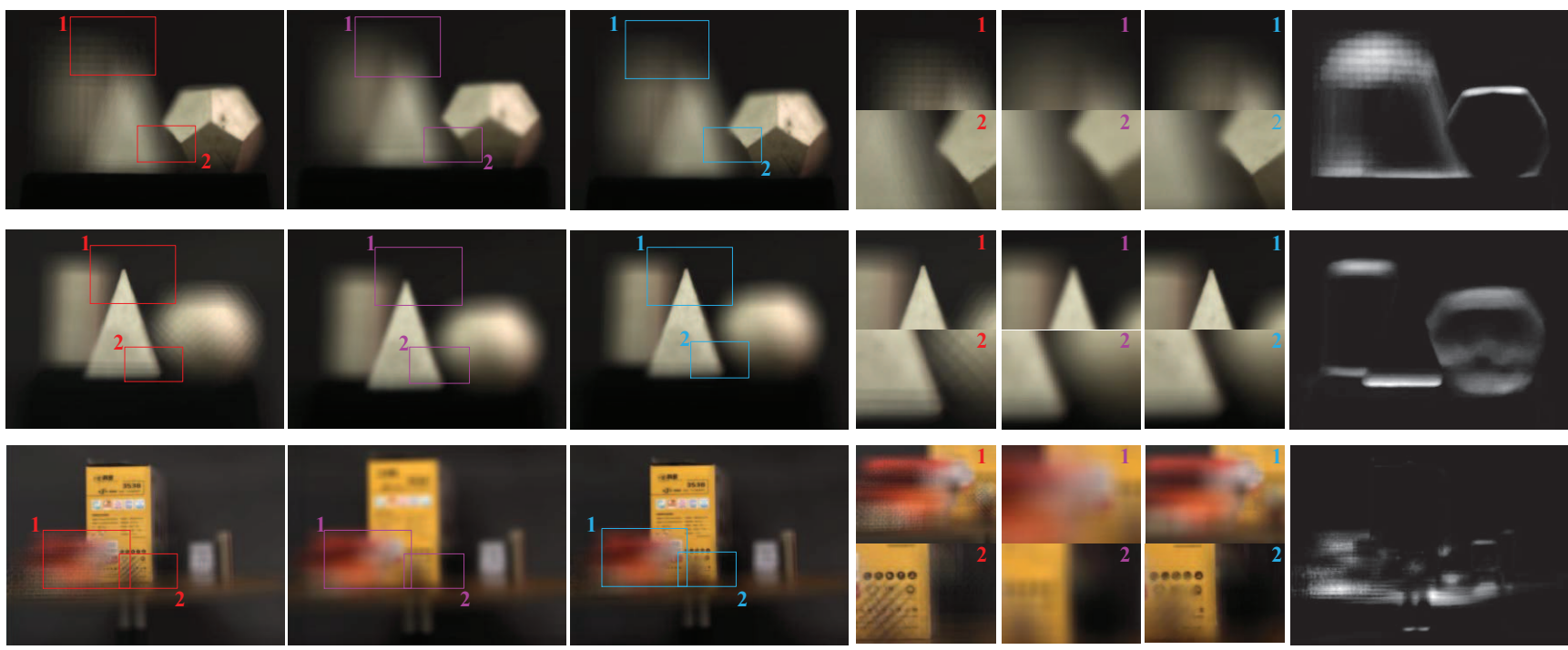

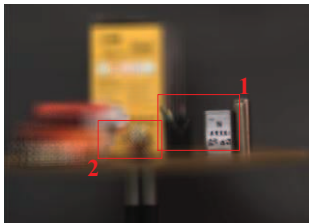

(a)

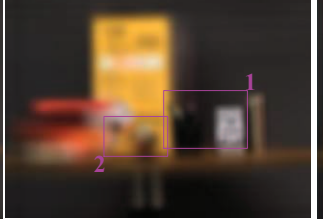

(b)

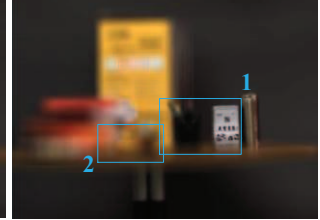

(c)

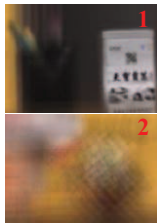

(d)

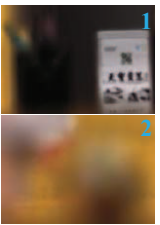

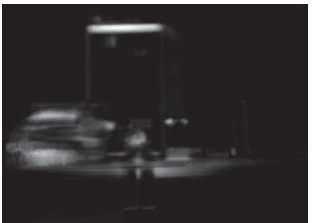

(e)

Figure 7. Results on the real light field camera array. (a) shows traditional light field refocusing results. (b) shows the refocusing results using prefiltered light fields. (c) shows our results. (d) shows the closeup views on the details. (e) shows the detected aliased regions.

sampling rates are much higher in these light field cameras than the camera array, a large $\gamma$ can be applied for aliasing detection and reduction. We also plan to estimate the relevant parameters adaptively and to accelerate our algorithm with parallel programming.

Acknowledgement. The work is supported by NSFC fund (61272287), “863” project (2012AA011803), Specialized Research Fund for the Doctoral Program of Higher Education (20116102110031), research grant of State Key Laboratory of Virtual Reality (BUAA-VR-12KF-02), China.

\section{References}

[1] E. H. Adelson, C. H. Anderson, J. R. Bergen, P. J. Burt, and J. M. Ogden. Pyramid methods in image processing. $R C A$ engineer, 29(6):33-41, 1984. 5

[2] T. Bishop and P. Favaro. The light field camera: Extended depth of field, aliasing, and superresolution. IEEE TPAMI, 34(5):972-986, 2012. 2

[3] M. Broxton, L. Grosenick, S. Yang, N. Cohen, A. Andalman, K. Deisseroth, and M. Levoy. Wave optics theory and 3-D deconvolution for the light field microscope. Optics express, 21(21):25418-25439, 2013. 1

[4] J.-X. Chai, X. Tong, S.-C. Chan, and H.-Y. Shum. Plenoptic sampling. In ACM SIGGRAPH, 2000. 1, 3

[5] Y. Ding, J. Yu, and P. F. Sturm. Multiperspective stereo matching and volumetric reconstruction. In ICCV, 2009. 2
[6] T. Georgiev and A. Lumsdaine. Reducing plenoptic camera artifacts. Computer Graphics Forum, 29(6):1955-1968, 2010. 2

[7] T. Georgiev, C. Zheng, B. Curless, D. Salesin, S. Nayar, and C. Intwala. Spatio-angular resolution tradeoffs in integral photography. In Eurographics Symposium on Rendering (EGSR), 2006. 2

[8] A. Isaksen, L. McMillan, and S. J. Gortler. Dynamically reparameterized light fields. In ACM SIGGRAPH, 2000. 1, 2

[9] A. K. Jain. Fundamentals of Digital Image Processing. Prentice Hall, 1989. 4

[10] N. Joshi, W. Matusik, and S. Avidan. Natural video matting using camera arrays. In ACM SIGGRAPH, 2006. 1

[11] C. Kim, H. Zimmer, Y. Pritch, A. Sorkine-Hornung, and M. Gross. Scene reconstruction from high spatio-angular resolution light fields. ACM TOG, 32(4):73:1-12, 2013. 1, 2

[12] A. Levin, W. T. Freeman, and F. Durand. Understanding camera trade-offs through a bayesian analysis of light field projections. In $E C C V, 2008.2$

[13] M. Levoy and P. Hanrahan. Light field rendering. In $A C M$ SIGGRAPH, 1996. 1, 2, 3, 6

[14] C.-K. Liang, G. Liu, and H. H. Chen. Light field acquisition using programmable aperture camera. In ICIP, 2007. 2

[15] C.-K. Liang, Y.-C. Shih, and H. Chen. Light field analysis for modeling image formation. IEEE TIP, 20(2):446-460, 2011. 3 

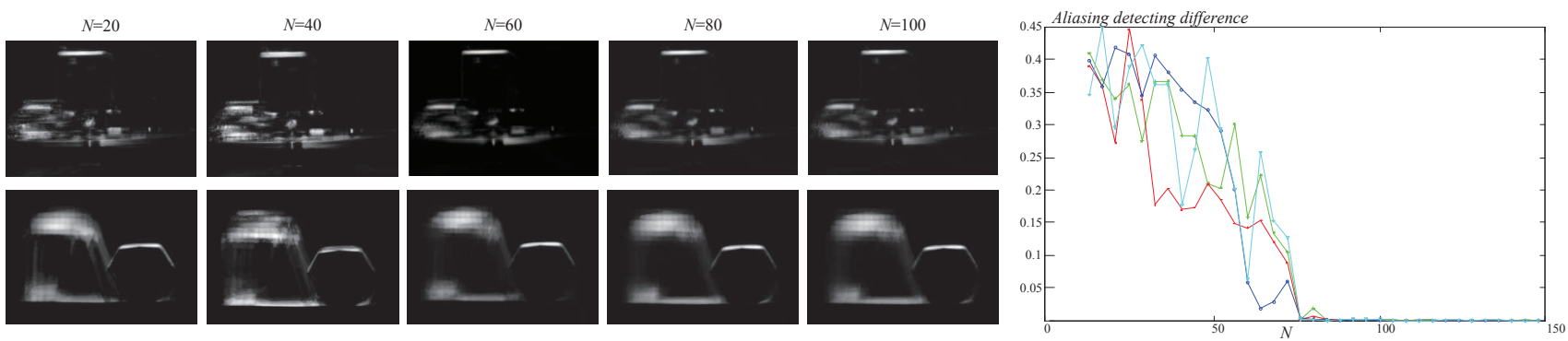

Figure 8. Aliasing detection using different $N$ s. Left: two sets of aliasing detection results with different $N$ s. Right: the plot shows the difference of the results using $N$ and $N-1$ vs. $N$.

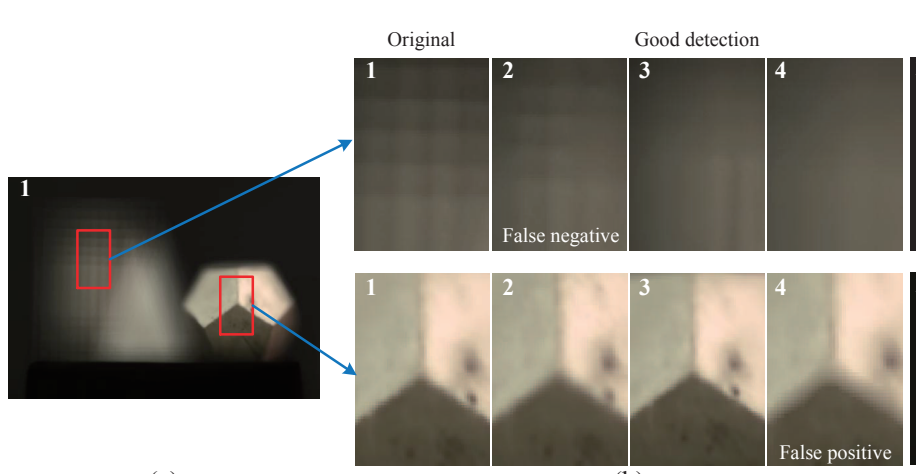

(a) (b)
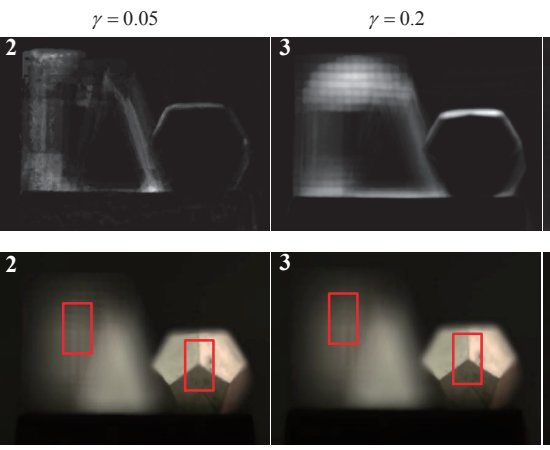

(c)

Figure 9. Aliasing detections using different $\gamma$ s. (a) shows a sample aliased image produced by light field refocusing. We highlight the aliased and aliasing-free regions in red. (b) shows the closeup views of the regions. (c) shows the aliasing detection and reduction results using different $\gamma$ s.

[16] A. Lumsdaine and T. Georgiev. Full resolution lightfield rendering. Indiana University and Adobe Systems, Technical Report, 2008. 2

[17] A. Lumsdaine and T. Georgiev. The focused plenoptic camera. In ICCP, 2009. 2

[18] Lytro. http://www.lytro.com. 1, 2

[19] K. Marwah, G. Wetzstein, A. Veeraraghavan, and R. Raskar. Compressive light field photography. In ACM SIGGRAPH Posters, page 49:1, New York, NY, USA, 2012. ACM. 1

[20] R. Ng. Fourier slice photography. In ACM SIGGRAPH, 2005. 1, 2

[21] P. Pérez, M. Gangnet, and A. Blake. Poisson image editing. In ACM SIGGRAPH, 2003. 5

[22] C. Perwaß and L. Wietzke. Single lens 3d-camera with extended depth-of-field. In SPIE, volume 8291, 2012. 2

[23] Raytrix. http://www.raytrix.com. 1, 2

[24] J. Stewart, J. Yu, S. J. Gortler, and L. McMillan. A new reconstruction filter for undersampled light fields. In Eurographics Symposium on Rendering (EGSR), 2003. 2

[25] V. Vaish. Synthetic aperture imaging using dense camera arrays. PhD thesis, Stanford University, CA, USA, 2007. 1, 2,5

[26] K. Venkataraman, D. Lelescu, J. Duparré, A. McMahon, G. Molina, P. Chatterjee, R. Mullis, and S. Nayar. Picam: An ultra-thin high performance monolithic camera array. $A C M$ TOG, 32(6):166:1-166:13, Nov. 2013. 1

[27] S. Wanner, C. Straehle, and B. Goldluecke. Globally consistent multi-label assignment on the ray space of $4 \mathrm{~d}$ light fields. In CVPR, 2013. 1, 2

[28] B. Wilburn, N. Joshi, V. Vaish, E. Talvala, A. Barth, A. Adam, M. Horowitz, and M. Levoy. High performance imaging using large camera arrays. ACM TOG, 24(3):765776, 2005. 1

[29] J. Yu and L. McMillan. A framework for multiperspective rendering. In Eurographics Symposium on Rendering (EGSR), 2004. 1

[30] X. Yu, R. Wang, and J. Yu. Real-time depth of field rendering via dynamic light field generation and filtering. Computer Graphics Forum, 29(7):2099-2107, 2010. 4, 6

[31] Z. Yu, X. Guo, H. Lin, A. Lumsdaine, and J. Yu. Lineassisted light field triangulation and stereo matching. In ICCV, 2013. 1, 2

[32] Z. Yu, J. Yu, A. Lumsdaine, and T. Georgiev. An analysis of color demosaicing in plenoptic cameras. In CVPR, 2012. 2

[33] M. Zwicker, W. Matusik, F. Durand, H. Pfister, and C. Forlines. Antialiasing for automultiscopic 3D displays. In ACM SIGGRAPH, 2006. 2 